Cahiers $d u$ MONDE RUSSE

\section{Cahiers du monde russe}

Russie - Empire russe - Union soviétique et États indépendants

$51 / 4 \mid 2010$

Sciences humaines et sociales en Russie à l'Âge d'argent

\title{
Heinrich Wölfflin en Russie
}

De la découverte de l'Italie et de l'art baroque russe à la conception de la méthode formaliste et structuraliste dans la critique littéraire

Heinrich Wölfflin in Russia: From the discovery of Italy to the conception of the Formalist and Structuralist methods in literary criticism

\section{Ekaterina Dmitrieva}

\section{(2) OpenEdition \\ Journals}

Édition électronique

URL : https://journals.openedition.org/monderusse/9205

DOI : 10.4000/monderusse. 9205

ISSN : $1777-5388$

Éditeur

Éditions de l'EHESS

Édition imprimée

Date de publication : 25 novembre 2010

Pagination : $507-520$

ISBN : 978-2-7132-2316-7

ISSN : $1252-6576$

Référence électronique

Ekaterina Dmitrieva, "Heinrich Wölfflin en Russie », Cahiers du monde russe [En ligne], 51/4 | 2010, mis en ligne le 20 décembre 2013, consulté le 03 septembre 2022. URL : http://journals.openedition.org/ monderusse/9205; DOI : https://doi.org/10.4000/monderusse.9205

Ce document a été généré automatiquement le 3 septembre 2022.

Tous droits réservés 


\section{Heinrich Wölfflin en Russie}

De la découverte de l'Italie et de l'art baroque russe à la conception de

la méthode formaliste et structuraliste dans la critique littéraire

Heinrich Wölfflin in Russia: From the discovery of Italy to the conception of the

Formalist and Structuralist methods in literary criticism

\section{Ekaterina Dmitrieva}

\section{NOTE DE L'AUTEUR}

Une version légèrement différente de cet article est parue en russe, E.E. Dmitrieva, "Genrih Vel'flin v Rossii : otkrytie Italii, barokko ili formal'nogo metoda v gumanitarnyh naukah? [Heinrich Wölfflin en Russie : découverte de l'Italie, du baroque ou de la méthode formaliste dans les sciences humaines?» in E.E. Dmitrieva, éd., Evropejskij kontekst russkogo formalizma ( $k$ probleme èstetičeskih perecečenij : Francija, Germanija, Italija, Rossija) [Le contexte européen du formalisme russe (De la question des croisements esthétiques : France, Allemagne, Italie, Russie)], M. : IMLI, 2009, p. 98-131.

1 L'image que l'on a de Heinrich Wölfflin peut être très diverse et le plus souvent fonction du domaine d'étude ou d'intérêt de chacun. L'historien d'art l'évoquera comme celui qui avait élaboré une méthode d'analyse des styles basée sur l'évolution de la perception visuelle; le critique littéraire se souviendra sans doute que son livre Die Kunstgeschichtlichen Grundbegriffe fut étudié par Boris Ejhenbaum à l'époque où il élaborait sa doctrine formaliste ; le spécialiste de la littérature russe du XVII siècle ou un dix-huitiémiste associera ce nom à la découverte du style baroque ${ }^{1}$. Pour d'aucuns encore, Wölfflin reste celui qui proposa une analyse pertinente de l'art italien de la Renaissance, à l'origine d'une nouvelle redécouverte de l'Italie autour de 1900, cependant qu'en Union soviétique, son nom était associé à la critique d'art bourgoise, considérée comme « méthodologiquement mauvaise ».

2 Aussi l'enjeu de cet article sera-t-il d'explorer l'itinéraire complexe de la réception et de l'appropriation russe de la théorie de Wölfflin dont l'emprise s'est affirmée, comme nous 
venons de le mentionner, dans différents domaines des sciences humaines telles l'histoire de l'art, l'histoire des littératures ou la théorie littéraire. d'une grande portée historique " (Nikolaj Punin) ${ }^{8}$. Une telle appréciation ne devait rien au hasard : dès les années 1910, on retrouve des marques de l'emprise de Wölfflin chez les critiques d'art russes. C'est ainsi que dans Obrazy Italii de Muratov, livre qui resta longtemps un guide intellectuel pour ceux qui voulaient se rendre en Italie ${ }^{9}$, l'empreinte de Wölfflin est repérable à plusieurs reprises. Muratov l'évoque dans le chapitre «Le baroque» comme celui qui a découvert l'art baroque de Rome: «Personne ne pourra plus percevoir le baroque comme le faisaient les contemporains de Stendhal : une suite mutilée et décadente de la Renaissance ${ }^{10}$. Même si Muratov ne cite pas toujours Wölfflin de manière explicite, le choix des époques, des artistes et des œuvres reste fortement influencé par sa pensée ${ }^{11}$.

7 Parallèlement au mérite d'avoir découvert le baroque italien à Rome, Wölfflin jouit à la même époque de la réputation de poète-historien. Ainsi, dans son article sur la valeur de l'esthétique, l'esthéticien Sezemann développe l'idée du lien nécessaire qui unit l'esthétique à l'art et, à ce propos, évoque Wölfflin: «Ce sont justement les meilleurs représentants de l'histoire de l'art, ceux qui ne sont pas simplement des archéologues, mais de vrais connaisseurs de l'art, qui deviennent nécessairement, dans leurs jugements stylistiques et dans leurs descriptions des œuvres d'art, de vrais poètes (comme par exemple Wölfflin dans L'art classique ou bien dans La Renaissance et le baroque. [...] Seul le mot poétique peut être congénial à l'impression que l'œuvre d'art produit sur nous). " ${ }^{12}$

En Russie l'intérêt pour l'œuvre de H. Wölfflin commença à partir des années 1910 et persista au-delà de la révolution, jusque dans les années 1930.

Allemagne (ou en Suisse) et leur traduction en Russie devenant insignifiant. Le premier ouvrage à être traduit fut son essai Die klassische Kunst (1898), qui parut à SaintPétersbourg en $1912^{2}$. Le livre Renaissance und Barock (1889) lui fit suite en $1913^{3}$, Die Kunstgeschichtlichen Grundbegriffe (1915) en 1930', Das Erklären von Kunstwerken (1921) en $1922^{5}$. Quant à son étude Italien und das deutsche Formgefühl (1932) [L'Italie et le sentiment allemand de la forme] elle fut traduite en russe presque aussitôt, en 1934, mais

l'importance méthodologique de l'ouvrage : « L'histoire du jugement esthétique au cours de ces trois dernières décennies était bien triste, [grâce à l'ouvrage de Wölfflin] on a enfin la possibilité de prendre conscience du problème des formes esthétiques et des lois du fonctionnement de l'œuvre d'art». On ne pourrait pas recommander d'autre ouvrage à quelqu'un qui « désire libérer son jugement esthétique du subjectivisme de sa propre opinion $»^{7}$.

La deuxième traduction de Wölfflin, celle de Renaissance und Barock, sortie sans aucun commentaire, fut néanmoins hautement appréciée par la critique comme un «livre 
qualifie de « formaliste malgré lui », dont les méthodes d'approche d'une œuvre d'art ne relèvent pas d'un dogme abstrait mais répondent aux besoins artistiques de l'époque: " On a inculqué au spectateur contemporain la fierté inouïe d'un créateur. [...] Wölfflin en sent tout le danger [...] Ce n'est pas aussi simple qu'on veut bien le dire, il n'est pas donné à chacun de pouvoir voir ce qui est véritablement. L'interprétation de l'œuvre d'art doit constituer une partie nécessaire de la formation artistique ${ }^{13}$.

De même B.L. Bogaevskij, un autre historien d'art, dont le but était à vrai dire de démontrer l'essence formelle de toute critique d'art: ce ne sont que les «formes de la vue » (formy smotrenija) qui constituent son seul et unique objet. On dirait que Wölfflin pourrait servir de preuve parfaite à une pareille thèse. Et pourtant... Il se trouve que ce que Bogaevskij apprécie plus particulièrement chez lui, ce n'est pas la méthode formelle mais les mécanismes psychologiques qui s'en dégagent : la formule "à cinq chaînons " de Wölfflin, ses cinq fameuses oppositions, ayant pour base « le processus psychologique de la vision sensible d'un objet » qui remonte à l'« œil qui palpe » de Herder ${ }^{14}$.

Une autre tendance de la psychologisation dans la méthode formelle de Wölfflin se manifesta dans la monographie collective Barokko v Rossii parue à Moscou en 1926 et qui, à vrai dire, réunissait des interventions faites aux séminaires de la Section des arts de l'espace de l'Académie d'État des sciences de l'art ${ }^{15}$. Le nom de Wölfflin y est évoqué quasiment par tous les coauteurs dont I.I. Brunov, G.V.Židkov, V.V. Zgura. Leur objectif commun était de démontrer l'existence du baroque russe comme une des modalités « du grand style universel ». En citant abondamment des passages tirés de Renaissance und Barock, déjà traduit à l'époque, et de Die Kunstgeschichtlichen Grundbegriffe qui attendaient encore leur traduction (ce qui montre par ailleurs que Wölfflin était suffisamment lu également en allemand), les jeunes critiques russes arrivaient à prouver, grâce au recours à tel ou tel indice formel proposé par Wölfflin, l'appartenance d'un monument architectural russe au style baroque ${ }^{16}$. Mais à y regarder de près on remarque qu'une des oppositions de Wölfflin, celle du linéaire et du pittoresque apparaît dans le livre plus fréquemment que les autres. Selon l'avis des auteurs, c'est notAmment le pittoresque "propre à l'originalité (samobytnost') russe» qui aurait préparé le terrain "pour l'épanouissement de certains principes du baroque » en Russie. Ainsi introduisent-ils dans une des oppositions formelles de Wölfflin une composante psychologique bien imprécise : la passion pour le pittoresque comme trait général du caractère russe ${ }^{17}$.

Dans les années 1910-1920, la critique littéraire commence aussi à être attirée par la méthode wölfflinienne de l'analyse des formes ${ }^{18}$. C'est ainsi qu'en 1919 Boris Ejhenbaum, un des fondateurs de l'école formaliste russe, lit attentivement les Concepts fondamentaux de l'histoire de l'art, ce dont témoigne une entrée dans son journal en date de janvierfévrier 1919: «Je continue Wölfflin. Constamment apparaissent des analogies avec la construction de l'histoire de la littérature. Il faudrait établir un système de concepts fondamentaux et soumettre la littérature à l'analyse, en regardant un phénomène donné à chaque fois d'un seul point de vue, en extrayant tout ce qui s'y rapporte. $»^{19}$

Évidemment ce n'est pas un hasard si Ejhenbaum cita par la suite Wölfflin dans son article " Théorie de la méthode formaliste » (1925), présenté au départ sous la forme d'un exposé qui souleva de fortes discussions. La démarche de Wölfflin lui paraissait alors très importante pour la mise au point doctrinale et tactique de la méthode formaliste et il y 
recourait également afin de libérer la critique littéraire de la pression de ce qu'il appelait alors l'esthétique d'en haut et des théories " toutes prêtes ou qui se prétendaient l'être ". En défendant la rupture qui s'opérait alors entre la théorie esthétique et l'œuvre d'art, il précisait :

Cette rupture, surtout par rapport à l'esthétique, est un phénomène plus ou moins typique pour l'histoire contemporaine de l'art. En mettant de côté nombre de problèmes d'ordre général (les questions de la beauté, de la finalité de l'art), l'histoire de l'art s'est concentrée sur des problèmes concrets. De nouveau on a mis à l'ordre du jour, en dehors de tous les critères esthétiques, la question de la compréhension de la forme artistique et de son évolution. [...] Des slogans caractéristiques sont apparus, tel celui de Wölfflin invoquant une histoire de l'art sans artistes...20

Deux années plus tard, Viktor Žirmunskij soumit lui aussi à une analyse approfondie la doctrine de Wölfflin dans son article « Les nouvelles tendances de la critique littéraire en Allemagne ». En décernant à Wölfflin le rôle d'un des maîtres « de la nouvelle génération des philologues (!) allemands ", il l'inscrit en même temps dans la lignée de penseurs et philosophes, tels Goethe, August et Friedrich Schlegel, qui commencèrent autour de 1800 à s'attaquer à la question de la " typologie des styles artistiques $»^{21}$.

Pourtant, à observer la question de près, il existait déjà, dès le début, une certaine discordance entre les formalistes russes et la méthode de Wölfflin. Elle se profila en $1925 \mathrm{chez}$ Ejhenbaum. Et si le ton général de son article "Théorie de la méthode formaliste » se présentait plutôt comme apologétique à l'égard de Wölfflin (ce n'est que dans une note en bas de page qu'Ejhenbaum mentionne comme incidemment que la théorie de Wölfflin aurait engendré en Allemagne un mouvement esthétique hostile à ses intentions de départ ${ }^{22}$ ), une nouvelle entrée dans son journal laisse comprendre toutes les raisons de son conflit avec «la science allemande ", qui ne fera que s'accentuer dans les années suivantes: "J'ai une drôle d'impression - comme si les Allemands étaient en retard par rapport à nous. Ils se démènent avec de vieux problèmes, très peu utiles ${ }^{23}$. Une dizaine de jours plus tard il précise : « Comme c'est différent chez eux! Mouvement esthétique $»^{24}$.

Il semble qu'il y avait également une autre raison à la divergence entre les formalistes russes et Wölfflin. Tandis que les premiers, selon l'avis de I. Čečot, avaient toujours en vue l'analyse d'une œuvre concrète, Wölfflin ne visait que les styles dans leur essence. Le concept de procédé (priëm) ainsi apparu, et particulièrement important chez Ejhenbaum et Šklovskij, ne l'avait jamais vraiment interessé25.

16 Force est de constater que la contestation de la méthode formaliste qui s'accentua en Russie vers la fin des années 1920 recourait souvent, elle aussi, à la méthodologie de Wölfflin (bien que le nom du critique d'art suisse pût ne pas être évoqué de façon explicite). Selon Medvedev, qui partit en guerre contre la méthode formaliste d'Ejhenbaum, celui-ci n'aurait pas pris en considération la position spécifique du formalisme européen qui se situait entre l'esthétique idéaliste en amont et l'esthétique naturaliste en aval. « Les formalistes européens ont combattu cette dernière encore plus que la première. [...] Le formalisme européen n'est pas indifférent aux questions idéologiques, ce qui ne l'empêche pas d'aspirer à une méthode concrète dans l'étude de la construction artistique $»^{26}$.

17 C'est ainsi qu'en assignant au formalisme wölfflinien une dimension idéologique (voire sociologique), Medvedev se rapprocha considérablement de l'optique de Vipper et de 
Sezemann, ceux-ci considérant Wölfflin comme un formaliste malgré lui, un formaliste pour qui le contenu valait autant que la forme.

Quant à Bahtin, à qui longtemps on attribua La méthode formelle dans la critique littéraire, lui aussi témoignait d'une certaine ambiguïté vis-à-vis de Wölfflin. En effet, même s'il remettait en cause l'idée d'histoire de l'art sans artiste, il était loin de renier toutes les possibilités de l'analyse typologique appliquée à la structure du texte. Ce qui lui répugnait pourtant dans cette théorie, c'est qu'elle considérait toute structure comme abstraite et impersonnelle. Selon Bahtin, les textes, à la différence des langues, sont intraduisibles pour la simple raison qu'« intraduisibles et encore moins réductibles à une conscience générale sont les personnalités qui les créent». De là vient l'idée bakhtinienne de la dépersonnification du texte comme " oubli de tout ce qui est lié aux relations dialogiques ${ }^{27}$.

En même temps, le concept primordial de la théorie esthétique de Bahtin mais également de son éthique - je pense à celui d'architectonique (arhitektonika) - remonte en grande partie aux idées avancées par Wölfflin dans sa thèse Prolégomènes à une psychologie de l'architecture (1886) où il a su réunir deux conceptions du terme. La première, dite kantienne, définit l'architectonique comme « système de la raison pure » - une entité qui contient à la fois son but et sa forme. La seconde commença à se répandre autour de 1900, initiée plus spécialement par le livre de Hildebrandt, Problèmes de formes dans l'art figuratif, traduit en russe en 1914. Ce dernier désignait l'architectonique comme être autonome de l'œuvre d'art conçu comme enjeu constructif du créateur. En reliant l'achitectonique kantienne à la conception de l'architectonique dans les arts figuratifs proposée par Hildebrandt, Wölfflin l'avait désignée dans sa thèse comme « essence des unités diverses dont la nécessité se définit par l'organisme et l'harmonie, centrés autour d'un individu humain ${ }^{28}$.

C'est précisement ce dernier point qui fut extrêmement précieux pour Bahtin qui, lui aussi, défendait la thèse selon laquelle l'architectonique ne pouvait exister que liée à un être humain. Pour lui l'architectonique se présentait comme "l'essence d'un monde anthropocentrique, non pas un système intellectuellement construit, mais une forme de vie en tant que telle ». C'est ainsi qu'en 1924, en parlant du caractère systématique de tout phénomène culturel, de l'appartenance de tout procédé artistique à l'unité de la culture, Bahtin pensait entre autres en catégories de l'esthétique wölfflinienne ${ }^{29}$.

Une nouvelle étape de la réception russe de Wölfflin, qui pourrait être désignée comme tentative marxiste ou quasi-marxiste de sa relecture, commence dans les années 1930. Je pense avant tout à deux longs articles, celui de R. Pel'še et celui de L. Rempel rédigés comme préfaces aux éditions russes des Concepts fondamentaux de l'histoire de l'art et de L'art de l'Italie et de l'Allemagne à l'époque de la Renaissance dont la fonction était de " faire passer " ces deux livres à travers la censure soviétique qui leur trouvait défauts et qualités en parts égales.

Pel'še, déjà connu comme un des co-rédacteurs du recueil Komsomol, na front iskusstva $!^{30}$, commença son introduction par une thèse conciliante : bien que Wölfflin soit formaliste, "ses œuvres sont d'une grande importance pour nous " ${ }^{31}$. Puis il essaya de prendre la méthode de Wölfflin comme prétexte pour entrer en guerre contre l'approche sociologique «fortement combattue par Lenin et par Plehanov » : «dans le domaine du 
contenu de l'art, le marxisme se sent fort, on possède les œuvres dites classiques, celles de Plehanov, de Mehring, certaines thèses de Marx, d'Engels et de Lenin, [mais] c'est toujours l'esthétique marxiste qui nous manque ». Sa tâche, en tant que préfacier, était donc de donner une réponse dialectique et marxiste aux questions soulevées dans le livre de Wölfflin, « corriger la méthode de Wölfflin par notre connaissance de la dialectique marxiste des relations entre le fondement et la superstructure ${ }^{32}$.

Rempel, publiciste révolutionnaire dans sa jeunesse ${ }^{33}$ qui commença son activité d'historien d'art dans les années 1930 et se spécialisa plus particulièrement dans les domaines de l'art du Caucase et de la critique de l'art bourgeois ${ }^{34}$, se montra dans sa préface beaucoup plus arrogant vis-à-vis de Wölfflin. À le lire, Wölfflin était, le "prédecesseur de la science fasciste bourgeoise", celui qui avait défini les traits caractéristiques de l'art allemand repris par la suite par les nationaux-socialistes : « Les théoriciens formalistes, sans se mêler officiellement de politique, ont de nouveau commencé à créer des théories de l'esprit national dans l'art : ils ont de nouveau sollicité l'esprit irrationnel de l'art gothique pour attaquer les fondements matérialistes de la Renaissance $»^{35}$.

Ce qui est néanmoins étonnant dans cette introduction c'est que, malgré son caractère visiblement simpliste et idéologiquement marqué, Rempel essaya d'inscrire Wölfflin dans la tradition néokantienne. Plus encore, il reprit la logique de Wölfflin des années 1910 quand celui-ci, dans ses ouvrages, avait transformé l'oppposition entre le baroque et la Renaissance en opposition entre l'Allemagne et l'Italie. Pourtant l'enjeu de Rempel fut autre: celui de protéger l'art matérialiste italien de l'art irrationnel du baroque allemand.

L'autre côté curieux de cette préface, comparée à celle de Pel'še, consiste dans le fait que si celui-ci, comme on a eu l'occasion de le mentionner, suggérait au lecteur soviétique de lire Wölfflin à travers Plehanov, maintenant c'était Plehanov lui-même qui se trouvait accusé par le préfacier d'avoir contribué par sa propre méthode à l'assimilation de « l'histoire de l'art bourgeois, y compris celle de Hildebrandt, Riegl et celle de Wölfflin » en Union Soviétique.

26 La conclusion de Rempel est tout de même rassurante : "Comment lire un formaliste ? Comment lire un formaliste intelligent ? On doit découvrir derrière le système idéaliste de Wölfflin certains traits rationnels et apprendre à les remettre sur leurs pieds $»^{36}$. Et pourtant ce dernier livre eut une mauvaise critique. V. Pavlov, lui-même co-traducteur de l'ouvrage, se mit à expliquer, en le reprenant presque mot à mot, le propos de Lunačarskij lors de son intervention au congrès international philosophique à Oxford en 1930 : toute la doctrine de Wölfflin pourrait facilement être réduite à ce que la doctrine marxiste enseignait depuis longtemps, le développement de la société humaine du féodalisme vers le capitalisme ${ }^{37}$.

Chose étonnante, aussi stimulante que fût la doctrine de Wölfflin tant pour les historiens d'art que pour les critiques littéraires, et malgré toutes les controverses propres à sa relecture en Russie dans les années 1910-1920, les historiens d'art se montrent critiques et presque hostiles vis-à-vis de Wölfflin à partir des années 1930. Même en laissant de côté les propos conjecturaux et idéologiquement marqués comme ceux de Pel'še ou de 
Rempel, restent néanmoins des chercheurs "sérieux", réputés, loin de tous les conjectures idéologiques qui se montrent visiblement critiques.

Je pense entre autres au philosophe et historien d'art V. Zubov, lié dans les années 1920 à l'Académie d'État des sciences de l'art (de même que Brunov, Židkov, Zgura déjà évoqués) dont la thèse d'État soutenue en $1946^{38}$ cite abondamment le nom de Wölfflin, mais chaque fois dans un contexte polémique. Il semble qu'il lui soit globalement reproché d'avoir négligé les contradictions propres à la Renaissance en tant que telle, et d'avoir trop schématisé et instrumentalisé les cinq "fameuses » antithèses stylistiques (linéaire / pittoresque, espace / profondeur, forme fermée / forme ouverte, unité / pluralité, clarté / dispersion), d'où « le caractère inévitablement statique de ses exégèses, l'impossibilité de concevoir les phénomènes dans leur genèse et leur dynamique ${ }^{39}$. Seul le chapitre " L'œil et le problème de l'optique artistique » dans les Concepts fondamentaux de l'histoire de l'art attire Zubov par la tentative pourtant bien dissimulée de déborder la méthode formaliste pour déboucher sur « l'étude historique concrète des formes de la vision du monde, socialement variables » :

Dans ses Concepts fondamentaux de l'histoire de l'art, Wölfflin prétend que tout peintre est lié par des possibilités optiques propres à son époque. [...] Tout n'est pas toujours possible. La vue a sa propre histoire et la découverte de ces strates optiques doit être envisagée comme tâche primaire de l'histoire de l'art. ${ }^{40}$

Ce dernier jugement rejoint en fait celui de Bogaevskij qui, dans les années 1920, valorisait le potentiel psychologique de la théorie formaliste de Wölfflin.

C'est aussi Alpatov, l'historien d'art sans doute le plus prisé à l'époque soviétique (du moins officiellement) qui, dans ses Études sur l'histoire universelle des arts, entre en polémique avec Wölfflin afin de donner «l'image de l'art classique de la Haute Renaissance» qui dépasse considérablement ce que lui-même apelle le dogme wölfflinien ${ }^{41}$.

V. Graščenkov est un autre cas de figure. Historien d'art d'une génération postérieure, dans sa postface à une nouvelle réédition des Images de l'Italie de Muratov, il attribue « les côtés faibles de sa description par ailleurs très forte » justement à l'influence de Wölfflin qu'il qualifie de « raisonnable » et de « dogmatique » :

C'est seulement sa caractérisation de la Renaissance romaine qui a souffert de la froide stérilité de la conception de l'art classique proposée par Wölfflin, ce dernier ayant influencé Muratov. Et si on ne peut pas nier que le formalisme de Wölfflin a aidé Muratov à devenir un critique d'art professionnel, avouons qu'il était pourtant de nature trop artiste pour ne pas vouloir franchir les bornes de la méthode wölfflinienne : Muratov ne pouvait ignorer les valeurs spirituelles de l'art. ${ }^{42}$

À côté du rôle que l'œuvre de Wölfflin a joué, tant dans la découverte de l'Italie en Russie au début $\mathrm{du} \mathrm{xx}^{\mathrm{e}}$ siècle que dans la conception de la méthode formaliste, il reste encore une dimension incontournable dès qu'on parle de sa réception en Russie. En effet, tant en Allemagne ${ }^{43}$ qu'en Russie, sa doctrine est devenue une base méthodologique pour la construction de la théorie et de l'histoire du baroque littéraire.

Pourtant il faut se garder des exagérations. Paradoxalement, on trouve en Russie le tout premier emploi du terme de baroque bien avant la parution de La Renaissance et le baroque de Wölfflin et même avant le Cicerone de J. Burckhardt (1855) auquel on attribue le mérite d'avoir employé pour la première fois le mot pour désigner le style de l'époque. En 
Russie, dès 1852, l'historien N.M. Snegirev définit l'église de la Trinité du village de Troicko-Lykovo comme "du style Renaissance ou pour mieux dire du style baroque apparu à Moscou vers la fin du XVII e siècle $»^{44}$. Par la suite, le terme fut employé par L. Dal' en $1873^{45}$, il apparaît dans l'entrée « Architecture» du dictionnaire de Brockhaus et Efron $^{46}$. On prétend ${ }^{47}$ que le terme acquiert (sous sa forme masculine barok) le droit de cité grâce à Sultanov, auteur du compte rendu de l'ouvrage de l'académicien A.M. Pavlinov, Histoire de l'architecture russe (1895). Tandis que celui-ci désignait le dernier quart du XVII e siècle comme " période de déclin ", Sultanov lui donna le nom d'" époque du baroque $»^{48}$. Ce n'est qu'en 1926 que le baroque architectural en Russie fut explicitement conçu, comme cela a déja été mentionné, à travers la théorie de Wölfflin, je pense notamment à la monographie collective Le baroque en Russie ${ }^{49}$.

En ce qui concerne le domaine de la critique littéraire, les premiers à employer le terme furent dans les années 1930 Dmitrij Čiževskij et Lev Pumpjanskij. Pourtant il ne s'agissait pas encore du baroque littéraire russe, Čiževskij ayant commencé à étudier la littérature baroque tchèque et ukrainienne ${ }^{50}$ et Pumpjanskij appliquant le terme à la littérature allemande ${ }^{51}$. Et c'est aussi Viktor Šklovskij qui, spontanément, qualifia les auteurs des années 1920 de " gens du baroque $»^{52}$.

Il a fallu attendre encore presque deux décennies pour que le médiéviste Igor Eremin applique de nouveau le terme à la littérature russe, notamment à la poésie de Simeon Polotskij ${ }^{53}$. Le nom de Wölfflin n'y apparaît toujours pas, mais on peut facilement relever l'écho de certaines de ses idées ${ }^{54}$. L'article avait suscité une telle discussion idéologique et même la persécution de son auteur, accusé alors de servilité vis-à-vis de la "science bourgEoise » que celui-ci n'osa plus reprendre le terme de baroque ni dans l'édition critique de Polockij de 1954 (collection "Literaturnye pamjatniki») ni dans ses nombreux articles consacrés à l'étude de la littérature russe du XVII siècle.

La situation, pourrait-on penser, changea considérablement en 1958, époque du dégel, lors $\mathrm{du} \mathrm{IV}^{\mathrm{e}}$ Congrès international des slavisants à Moscou. La question générale posée à tous les participants était la suivante : «Y avait-il une "littérature dite du baroque" dans les pays slaves? » Pourtant, la réponse de la plupart des participants fut négative, ceuxci optant pour le terme de préclassicisme. Le seul à s'opposer au scepticisme ambiant visà-vis du baroque fut de nouveau Eremin ${ }^{55}$. Quelques années plus tard, le fameux article du dix-huitiémiste Aleksandr Morozov, "La question du baroque dans la littérature russe du XVII ${ }^{e}-X V I I{ }^{e}$ siècle», fut accueilli avec la même indignation par la critique soviétique officielle ${ }^{56}$.

À notre connaissance, le premier à avoir appliqué certaines idées de Wölfflin à l'étude du baroque littéraire russe fut Aleksandr Pančenko qui, en 1974, cita un passage de La Renaissance et le baroque pour prouver le caractère baroque de la poésie de Simeon Polockij ${ }^{57}$, ce même Polockij, déjà héros de l'article de Eremin cité plus haut.

Les années 1970-1980 se présentent en général comme très propices à une nouvelle relecture de Wölfflin et ceci surtout dans le domaine de la critique littéraire. On voit son nom apparaître dans des manuels universitaires ${ }^{58}$, dans des études consacrées à la littérature (culture) du XVII ${ }^{e}$, XVIII ${ }^{59}$ et même du début du Xx $x^{e}$ siècle ${ }^{60}$. Enfin la première thèse à étudier l'œuvre de Wölfflin fut soutenue à l'université de Leningrad ${ }^{61}$.

39 La publication, à Tartu en 1976, du livre d'Igor Černov sur la théorie littéraire marqua l'apogée de cet intérêt ${ }^{62}$. Son enjeu était, comme en témoigne déjà le titre, de fournir une 
théorie du baroque littéraire s'appuyant sur la méthode de Wölfflin en l'appliquant par la suite à l'étude de la littérature russe des XVII ${ }^{\mathrm{e}}$ et XVIII ${ }^{\mathrm{e}}$ siècles.

Il est surprenant de voir à quel point Černov, qui voulait montrer historiquement comment Wölfflin avait su réhabiliter le baroque, à la fois comme style et comme concept, procéda lui-même de façon semblable, mais dans un contexte qui n'était plus le même. Disciple de Jurij Lotman, représentant de l'école sémiotique de Tartu, Černov était peut-être le mieux placé pour concevoir toute l'ampleur de la méthode formaliste qui était à la base de la démarche de Wölfflin, à laquelle il attribua le nom de paradigmatique baroque. Cependant, ne se contentant pas de cette méthode formaliste, il chercha à la compléter par d'autres dimensions: la dimension sémantique de Pitirim Sorokin permettant de penser le baroque dans le contexte idéologique et philosophique et la dimension syntactique de A. Hauser permettant de situer le baroque dans l'histoire sociale de l'Europe.

Černov ne fit pas que « compléter » Wölfflin par Sorokin et Hauser, il donna également à sa théorie une dimension psychologique, à vrai dire absente chez Wölfflin mais que les lecteurs russes étaient toujours soucieux de lui attribuer (je pense notamment à Bogaevskij, Zgura, Bahtin, Zubov). Wölfflin, prétend Černov, "introduit des caractéristiques psychologiques dans la méthode formelle au moment où il essaie de cataloguer le monde extérieur en suivant des principes d'expression empruntés au corps humain $»^{63}$.

La boucle semble bouclée : la méthode formelle de Wölfflin, qui suscita en Russie de l'intérêt tantôt pour le baroque italien, tantôt pour le baroque russe ou bien pour l'approche formaliste dans les études littéraires, se trouve revendiquée dans les années 1970, cette fois-ci dans le cadre de l'approche sémiotico-structurelle concernant les problèmes du baroque russe. La doctrine de Wölfflin elle-même s'y retrouve " humanisée », tournée vers l'homme et le collectif humain, ce qu'on pourrait désigner au préalable comme trait distinctif de sa relecture russe ${ }^{64}$.

Il s'agit là du meilleur moment de la réinterprétation de Wölfflin en Russie suivi par deux tentatives fort remarquables, celles de Mikhail Lifšic et de Ivan Čečot, de trouver à Wölfflin des analogues russes ayant les mêmes qualités mais échappant aux points faibles propres à la doctrine de l'historien d'art suisse-allemand. Ces deux sosies russes sont les deux philosophes religieux du début du xx $x^{e}$ siècle, Ivan Il'in ${ }^{65}$ et Pavel Florenskij ${ }^{66}$.

\section{NOTES}

1. Cf. L.I. Sazonova, Literaturnaja kul'tura Rossii : Rannee Novoe vremja [La culture littéraire de la Russie : le tout début des temps nouveaux], M. : Jazyki slavjanskoj kul'tury, 2006, P. 17.

2. Genrih Vel'flin, Klassičeskoe iskusstvo: Vvedenie v izučenie italjanskogo Vozroždenija [Heinrich Wölfflin, L'Art classique : Introduction à l'étude de la Renaissance italienne], trad. de l'allemand par A.A. Konstantinova, V.M. Nevežina, SPb. : Brockhaus-Efron, 1912. 
3. Genrih Vel'flin, Renessans $i$ barokko [Heinrich Wölfflin, Renaissance et baroque], trad. de l'allemand par E. Lundberg, M. : Grjaduščij den', 1913.

4. Genrih Vel'flin, Osnovnye ponjatija istorii iskusstv: Problema evoljucii stilja $v$ novom iskusstve [Heinrich Wölfflin, Les principes fondamentaux de l'histoire de l'art: le problème de l'évolution du style dans l'art moderne], trad. de l'allemand par A.A. Frankovskij, M. : Academia, 1930.

5. Genrih Vel'flin, Istolkovanie iskusstva [Heinrich Wölfflin, Réflexion sur l'histoire de l'art], trad. et préface de B. Vipper, M. : Delfin, 1922.

6. Genrih Vel'flin, Iskusstvo Italii $i$ Germanii epohi Renessansa [Heinrich Wölfflin, L'art de l'Italie et de l'Allemagne à la Renaissance], trad. de l'allemand par L.I. Nekrasova et V.V. Pavlova, M. : OGIZ, 1934.

7. F. Zelinskij, «Predislovie k russkomu izdaniju [Introduction à l'édition russe]», in Genrih Vel'flin, Klassičeskoe iskusstvo, p. 3.

8. Severnye zapiski, n’ 2, 1914, p. 190.

9. Pavel Muratov, Obrazy Italii [Images d'Italie], M. : Izd. Z.I. Gržebina, 1911-1912, 3 vol.

10. Ibid., vol. 2, p. 104.

11. Cf. V.N. Grjaščenkov, « Posleslovie [Postface] », in Muratov, Obrazy Italii, M.: Galart, 1993, vol. 2, p. 303.

12. V.E. Sezemann, «Ėstetičeskaja ocenka v istorii iskusstva (K voprosu o svjazi istorii iskusstva s èstetikoj) [La notion d'esthétique dans l'histoire de l'art (De la question du lien de l'histoire de l'art avec l'esthétique)] », Mysl', nº 1, 1922, P. 117-147.

13. Vel'flin, Istolkovanie iskusstva, p. 5-6.

14. B.L. Bogaevskij, «Zadači iskusstvoznanija (Sovremennye problemy izučenija izobrazitel'nih iskusstv) [Connaissance de l'art (problèmes contemporains de l'étude des arts appliqués)]», Zadači i metody izučenija iskusstv: Stat'i [Étude des arts, questions et méthodes : Articles], SPb. : Academia, 1924, P. 45, 48.

15. A.I. Nekrasov, éd., Barokko v Rossii [Le baroque en Russie ], M. : Tipo-lit. Gos. Akad. hud. nauk, 1926.

16. Cf. I.I. Brunov, « $K$ voprosu o tak nazyvaemom russkom barokko [De la question du dit baroque russe]», in Nekrasov, éd., Barokko v Rossii, p.41-42; G.V.Židkov, « K postanovke problemy o russkom barokko [De la question du baroque russe] », ibid. p. 104.

17. Cf. V.V.Zgura, «Problema vozniknovenija barokko v Rossii [De l'apparition du baroque en Russie] », ibid. p. 11-42.

18. Voir à ce sujet A.A.Gornyh, "Ranneformalistskie i simvolistskie koncepcii hudožestvennoj formy [Conceptions préformalistes et symbolistes de la forme artistique] », Filosofskij poisk [Recherches philosophiques], $2^{\mathrm{e}}$ éd., Vitebsk, 1996, P. 96-110; I.D.Čečot, Osnovnye ponjatija istorii iskusstva Genriha Vel'flina: opyt issledovanija iskusstvovedčeskoj teorii $i$ metodologii [Les principes fondamentaux de l'histoire de l'art d'Heinrich Wölfflin : tentative d'élaboration d'une théorie de l'histoire de l'art et d'une méthode], exposé de thèse, L. : LGU, 1983, P. 3-4.

19. Cité d'après Boris M. Ejhenbaum, O literature [De la littérature], M. : Sovetskij pisatel', 1987, P. 511).

20. B. Ejhenbaum, «Teorija formal'nogo metoda [Théorie de la méthode formelle] », $O$ literature, p. 377). 
21. Viktor Žirmunskij, « Novejšie tečenija istoriko-literaturnoj mysli v Germanii [Les derniers courants de la pensée historique et littéraire en Allemagne] », Poètika, sbornik stat'ej. Vremennik otdela slovesnyh iskusstv [Poétique, recueil d'articles. Périodique du département des arts de la littérature], II., L., 1927, p. 6, 15.

22. Ejhenbaum, « Teorija formal'nogo metoda » p. 377.

23. Ibid., entrée du 13 janvier 1925, p. 511.

24. Ibid., entrée du 6 février 1925.

25. Čečot, Osnovnye ponjatija istorii, p. 34.

26. P.N. Medvedev, Formal'nyj metod $v$ literaturovedenii: kritičeskoe vvedenie $v$ sociologičeskuju poètiku [Méthode formelle en critique littéraire : introduction critique à une poétique sociologique, L. : Priboj, 1928).

27. M.M. Bahtin, «Problema teksta [La question du texte] », Sobranie sočinenij [Æuvres complètes], vol. 5, M., 1996, P. 628. Sur la question du rapport de Bahtin au « formalisme occidental », voir Roland Galharague, «Une ruse de la raison dialogique : Bahtin et le formalisme occidental », Cahiers du Monde russe et soviétique, 27 (3-4), 1986, p. 261-288.

28. Bahtin, Sobranie sočinenij, vol.1, Filosofskaja esstetika 1920-h godov [Esthétique philosophique des années 1920], M.: Russkie slovari : jazyki slavjanskoj kul'tury, 2003, P. 526 (voir le commentaire de V. Ljupunov).

29. Ibid.

30. R. Pel'še, Tarakanov, Čičerov, éds., Komsomol, na front iskusstva! Materialy pervogo vsesojuznogo soveščanija po hudožestvennoj rabote sredi molodeži [Komsomol, en avant sur le front de l'art! Documents de la première réunion fédérale sur le travail artistique chez les jeunes], Tehkinopečat', 1929.

31. Pel'še, « O faktorah razvitija iskusstva [Des facteurs du développement de l'art] », in Vel'lflin, Osnovnye ponjatija, p. VII.

32. Ibid., p. XVI.

33. Cf. L.I. Rempel', Krasnaja gvardija $v$ Krymu, 1917-1918 [La garde rouge en Crimée, 1917-1918], Simferopol : Krymgosizdat, 1931; Idem, Povstanci v Krymu [Les insurgés en Crimée], 1920.

34. cf. L.I. Rempel', "Nemeckij formalizm i ego fašistskie kritiki [Le formalisme allemand et les critiques fascistes ", Iskusstvo, n 3, 1934.

35. L.I. Rempel', «Genrih Vel'flin [Heinrich Wölfflin]», in Vel'flin, Iskusstvo Italii $i$ Germanii, p. 26.

36. Ibid, p. 52.

37. Akademija arhitektury, $\mathrm{n}^{\circ} 1-2,1934$, p. 144 . Cf. le propos de Lunačarskij : «Certes dans toutes ces conceptions, il y a une certaine dose de vérité, mais à quoi mène cette vérité ? À l'observation de mouvements existant réellement qui mènent du simple au compliqué, du stable et déterminé à l'instable et indéterminé, des efforts collectifs au libre individualisme. Mais ce mouvement n'est que le reflet nécessaire du développement du féodalisme au capitalisme de la société humaine ", A.V. Lunačarskij, " Novye tečenija v teorii iskusstva $\mathrm{v}$ Zapadnoj Evrope i marksizm [Nouveaux courants dans la théorie de l'art en Europe de l'Ouest et marxisme] » Sobranie sočinenij [Guvres complètes], (8 vol.), t. 8, M. : Hudožestvennaja literatura, 1967, p. 63-72 
38. Le texte de la thèse n'a été publié qu'en 2001, V.P. Zubov, Arhitekturnaja teorija Al'berti [La théorie architecturale d'Alberti], SPb.: Aletejja. Je tiens ici à remercier Ilona Svetlikova de m'avoir indiqué cette source.

39. Ibid., p. 34 ; cf. également p. 112-113, 342, 343, 344.

40. Ibid., p. 415.

41. Alpatov qualifie Andrea del Sarte, dont Wölfflin se servait pour étudier l'art de la Renaissance, d'artiste suffisamment «borné» pour pouvoir prétendre au statut d'exemple : «Et Wölfflin qui a dégagé avec une telle évidence les lois de l'art classique à partir de ses compositions se serait en vain efforcé de nous le présenter comme un des héros de la Haute Renaissance », M.V. Alpatov, Ėtjudy po vseobščej istorii iskusstv [Études sur l'histoire universelle des arts], M. : Sovetskij hudožnik, 1979, P. 113.

42. Grjaščenkov, « Posleslovie », t. 1, P. 305.

43. Cf. Klaus Garber, Zum Bilde Walter Benjamins, Munich: Wilhelm Fink Verlag, 1992, p. 234-236.

44. N.M. Snegirev, Russkaja starina v pamjatnikah cerkovnogo i graždanskogo zodčestva [La Russie d'antan dans l'architecture religieuse et civile], M., 1852, P. 23-24.

45. L. Dal', «Istoričeskoe issledovanie pamjatnikov russkogo zodčestva [Étude historique des monuments d'architecture russe] », Zodčij, n 5, 1873, P. 59.

46. Ėnciklopedičeskij slovar' pod red. I.E. Andreevskogo [Dictionnaire encyclopédique sous la réd. de I.E. Andreevskij], t. 2, SPb. : F.A. Brockhaus, I.A. Efron, 1890, P. 275.

47. Zgura, Problema vozniknovenija barokko v Rossii, P. 13.

48. N.V.Sultanov, Recenzija na sočinenija A.M. Pavlinova. Istorija russkoj arhitektury, drevnosti Jaroslavskie i Rostovskie, drevnie hramy Vitebska i Polocka i derevjannye cerkvi $g$. Vitebska [Compte rendu de l'ouvrage de A.M. Pavlinov, Histoire de l'architecture russe, des antiquités de Jaroslavl' et de Rostov, des temples antiques de Vitebsk et de Polock et des églises en bois de Vitebsk], SPb., 1897 (Tiré à part de « Otčet o XXXVII prisuždenijah nagrad grafa Uvarova [Rapport sur les 37 décernements de récompenses du comte Uvarov]»).

49. Nekrasov, Barokko $v$ Rossii.

50. Čiževskij lui-même parle des préoccupations baroques de sa jeunesse dans une revue qu'il rédigerait dans les années 1950. Cf.: D. Tchishevski, «Das Problem des slavischen Barocks ", Wissenschaftliche Zeitschrift der Ernst-Moritz-Arndt-Universitaet Greifswald, Jahrg. 6, 1956-1957 Gesellschafts- und sprachwissenschaftliche Reihe, P. 66-77.

51. L.V.Pumpjanskij, "Trediakovskij i nemecskaja škola razuma [Tredjakovskij et l'école rationnelle allemande] 》, Zapadnij sbornik, 1, 1930, P. 160.

52. « Gens de notre temps, gens du détail extrême, gens du baroque », Viktor Šklovskij, Poiski optimizma [Recherches d'optimisme], M., 1931, P. 114-115.

53. I.P. Eremin, «Poètičeskij stil' Simeona Polockogo [Le style poétique de Simeon Polockij] », Trudy Otdela drevnerusskoj literatury [Travaux du département de littérature russe ancienne], t. 6, M.-L., 1948, P. 125-153.

54. Ibid., p. 141, 137.

55. N.K.Gudzij, S.V.Nikol'skij, A.N. Robinson, éds., Sbornik otvetov na voprosy po literaturovedeniju. IV Meždunarodnyj s"ezd slavistov [Recueil des réponses aux questions de critique littéraire. IV ${ }^{\mathrm{e}}$ congrès des slavistes], M., 1958, P. 83. 
56. A.A. Morozov, «Problema barokko $\mathrm{v}$ russkoj literature XVII-načala XVIII veka (sostojanie voprosa i zadači izučenija) [La question du baroque dans la littérature russe du XVII -début du XVIII siècle] », Russkaja literatura, n 3, 1962, P. 34-38.

57. "Simeon, comme tous les hommes de grande culture du XVII ${ }^{e}$ siècle, se sentait l'heureux détenteur de la vérité. Wölfflin avait déjà observé ce trait de la culture baroque : "Le baroque était un sentiment propre de son droit exclusif à l'existence et de son infaillibilité exceptionnelle, comme n'importe quel autre style" ", A.M. Pančenko, «O smene pisatel'skogo tipa v petrosvkuju epohu [Du changement du style d'écriture à l'époque pétrine] », Problemy literaturnogo razvitija $v$ Rossii pervoj poloviny 18ogo veka [Les problèmes du développement littéraire en Russie au cours de la première moitié du XVIII ${ }^{\mathrm{e}}$ siècle], L., 1974, p. 115.

58. Cf. V.V.Kuskov, Istorija drevnerusskoj literatury [Histoire de la littérature russe ancienne], M., 1977, P. 290.

59. Cf. L.I. Tananaeva, « Portretnaja živopis' Karela Škrety [L'art du portrait selon Karel Škreta], in A.I. Rogov, A.V.Lipatov, L.A.Sofronova, éds., Slavjanskoe barokko. Istorikokul'turnye problemy epohi [Le baroque slave: questions historiques et culturelles de l'époque], M., 1979, P. 302.

60. I.P. Smirnov, «Barokko i opyt poètičeskoj kul'tury načala XX veka [Le baroque et l'expérience de la culture poétique du $\mathrm{xx}^{\mathrm{e}}$ siècle]", in Rogov, Lipatov, Sofronova, éds., Slavjanskoe barokko, p. 344.

61. Čečot, Osnovnye ponjatija istorii.

62. I.A.Černov, Iz lekcii po teoretičeskomu literaturovedeniju. 1. Barokko, literatura, literarurovedenie [Cours de théorie littéraire. 1. Baroque, littérature, critique littéraire], Tartu, 1976, P. 1-15.

63. Ibid., p. 58-59.

64. Cf. également l'article de Lotman, où il dit nécessaire de combiner dans l'étude du baroque la sémantique, la syntagmatique et la pragmatique, cette dernière étudiant la relation du signe au collectif humain ", Ju.M. Lotman, "Zamečanija k problème: barokko $\mathrm{v}$ russkoj littérature [Quelques remarques sur le problème du baroque dans la litterature russe] », Československá Rusistika, R. 13, n 1, 1968, p. 23.

65. Mihail Lifšic, $V$ mire éstetiki. Non finito [Dans le monde de l'esthétique. Non finito], M. : Izobr. iskusstvo.

66. I.D. Čečot, «Problema klassičeskogo iskusstva i barokko v rabotah G. Vel'flina o hudožnikah XVII i XIX vekov [L'art classique et le baroque dans les travaux de H. Wölfflin sur les artistes des XVII et xIX ${ }^{e}$ siècles] ", Problemy iskusstvozanija $i$ hudožestvennoj kritiki: Voprosy otečestvennogo i zarubežnogo iskusstva [Connaissance de l'art et critique artistique : questions sur l'art national et étranger], $2^{\mathrm{e}}$ éd., L. : Izd. Len. Universiteta, 1982, P. 51-109. 


\section{RÉSUMÉS}

Résumé

L'article montre comment la recherche que Wölfflin effectua dans le domaine de l'histoire de l'art, et notamment de l'art italien, avait mené en Russie à la découverte du baroque spécifiquement russe et contribua également à l'élaboration de la méthode formaliste dans l'étude littéraire. L'autre enjeu de l'article est d'étudier comment, dans la période soviétique, on a su approprier les idées de l'historien suisse à l'idéologie marxiste.

\section{Abstract}

The article shows how Wolfflin's research in the fields of history and art, and more particularly Italian art, led to the discovery in Russia of the specifically Russian Baroque and contributed to the elaboration of the formalist method in the study of literature. The article also studies how the Swiss historian's ideas were successfully appropriated by Marxist ideology during the Soviet period.

\section{AUTEUR}

\section{EKATERINA DMITRIEVA}

École normale supérieure, Paris, UMR 8547 équipe « Transferts culturels », Académie des sciences de Russie 